\title{
Toward a Central Repository for Sharing Nursing Informatics' Best Practices
}

Judith Effken, Charlotte Weaver, Kelly Cochrane, Ida Androwich, and Ann O-Brien

One of the most important findings to date of the Big Data Project initiated by the University of Minnesota is that nurses in 2016 are

\footnotetext{
"data-rich, information-poor . . . because each health care organization implements an EHR system without the ability to leverage lessons learned from organizations that have gone before them or access a "best practice" central repository that holds examples of data sets complete with clinical terms mapped to standardized terminologies such as clinical LOINC and SNOMED-CT" . ${ }^{1}$
}

Building on prior work done by the Transforming Nursing Documentation Working Group 10, in 2015 the authors volunteered to explore the feasibility of creating a central repository for best practices in nursing informatics. In this report, we share what we discovered.

We started our explorations by talking to several nurse leaders known to have faced the challenges of nursing documentation in their own settings and who could offer solutions that we hoped they might share. ${ }^{2}$ Obviously, there is no need for a best practices repository unless there are best practices to be shared and people willing to do so.

We interviewed Dr. Patricia Sengstack, who is CNIO (Chief Nursing Informatics Officer) at the Bon Secours Health System. Dr. Sengstack had led an evaluation of the patient admission assessment nursing documentation in her health system, during which she found that completing every field in the electronic nursing admission assessment alone required 532 key 
clicks including 14 different screening assessments. Overall the admission assessment had been configured so that there were 153 "required fields" that must be completed. Further study led Sengstack to estimate that only $25 \%$ of the nursing data in the electronic health record was actually useful to nurses. Bon Secours is now engaged in a streamlining process, identifying what is of little or no use-or simply a historical relic. She is also working with Bon Secours quality experts to assist in identifying if items in the assessment are truly required by CMS or the Joint Commission, or if the organization's interpretation mandates the documentation. Sengstack was willing to share the streamlining process that Bon Secours used with other organizations via a central repository.

We also interviewed Dr. Jane Englebright, CNO (Chief Nursing Officer) at HCA (Health Corporation of America). Engelbright and her colleagues have begun to tackle the burden of their own nursing documentation one problematic workflow at a time. First, a team of nurses develops what they view as the ideal workflow and then maps how relevant data should flow to better facilitate patient care. From these maps, the team drafts generic technical specifications for programmers. Because the specifications are generic, they work equally well for the two vendors that support HCA's electronic health records (EHRs). The magic of formulating generic specifications for nursing applications is that they become both vendor neutral and widely sharable among the nursing informatics community. Informatics nurses would profit widely from this exemplar, as well as the specifications themselves, so that they could either use the same specifications (another way of achieving standardization across the profession) or modify them as needed.

One of the challenges to achieving sufficient standardization so that we can use large national or international sets of nursing data for research is the fact that so few nurses know how to codify nursing data using SNOMED CT and LOINC. This lack of knowledge will become 
increasingly problematic as Federal regulations begin to require that nursing data utilize these coding systems. In a conversation with Drs. Judith Warren and Susan Matney, we learned that they are already sharing their knowledge of coding, including these resources on the National Library of Medicine website

\section{(https://www.nlm.nih.gov/research/umls/Snomed/nursing terminology resources.html). Based}

on the complexity of coding, it seems to us that such resources with exemplars would be extremely useful and should be housed in a central repository. In addition to the repository, however, we agreed that there should be a concerted effort to educate more nurse informaticists on coding. Without that skill, it will not be possible to achieve the goal of using national or international data sets as the basis for the research needed to improve nursing practice.

Clearly, there are best practice exemplars in our field and nurses are ready to share them. However, in our conversations, we also identified several potential barriers to achieving a central repository for nursing informatics best practices. The first challenge is finding a home for the repository. With the invaluable assistance of Rebecca Freeman at ONC and Kelly Cochran at ANA, we have begun to discuss creating a repository with federal agencies, colleges of nursing, and private companies. Housing the repository in a College of Nursing with an active informatics research program has numerous benefits because students could develop and manage the site, help curate the materials submitted, assist with the process of updating the materials as needed, and perhaps even use the data for their own research studies (in collaboration with the data owners). A successful example of a University-run repository is the Center for Health Policy at the University of New Mexico, which is a collaborative effort of the University with the Robert Wood Johnson Foundation (http://healthpolicy.unm.edu/about). Another example is provided by The National Center for Interprofessional Practice and 
Education (https://nexusipe.org/), whose site shares a variety of materials. A third example is the Virginia Henderson Library.

The second challenge is obtaining funding to build, maintain, and evaluate the site. We are investigating several grant possibilities and exploring potential partners to collaborate on this project, including ANI, ANA, CINAHL, NLM, and several graduate informatics programs. We have been advised by colleagues at NLM and AHRQ that having a viable sustainability plan is essential for funding, as is collaboration (e.g., including quality improvement, vendor, and management perspectives). Ensuring sustainability raises questions about long term financing of the repository. Certainly there is a potential financial benefit to using something someone else created because the time and effort required to create the same thing yourself is very high. Although free access to "exploring" the site is ideal, it might be necessary to assign fees for downloading materials_or require a "membership".

A third challenge is providing value (monetary or otherwise) to those groups sharing their best practices or research data. A nursing informatics researcher told us that she would welcome putting her decision support tool in a repository like this because she is having difficulty getting the validated instrument (currently in use locally in paper form) translated into an electronic version for use within the EHR. The organizations she is working with insist on hard coding the instrument within their vendor's system, but she wants it to be available for all to use. The researcher also wanted to be able to analyze the data collected via the instrument she developed. Providing a repository for nursing informatics research data seems not only reasonable, but critically important to improving nursing practice and patient health outcomes. However, accommodating research data for sharing purposes places additional constraints on repository design; and may be better served by a designing a separate warehouse. 
The urgent need for a repository of best informatics practices is clear. The burden of nursing documentation continues to grow, obscuring valuable information. The potential benefits of the EHR have yet to be realized in terms of decision support, communication, research, and improved patient safety and quality outcomes. Continuing to work in silos slows progress. Publishing our work is critical, but sharing vendor-neutral work products is likely to move the field ahead even faster. We must ensure that documentation innovations use standardized coding and are vendor neutral so the shared data from many settings can be analyzed and used to improve patient outcomes on a very large scale (something only "big data" can do). We intend to share what we have learned with a variety of audiences in the coming year including, not only the informatics community, but also nurse executives, hospital executives, and others. We will continue to pursue funding and partners to make the repository for nursing informatics best practices a reality. We welcome your feedback—and your help!

\section{Acknowledgements}

The authors gratefully acknowledge the continuing and invaluable assistance of Dr. Rebecca Freeman, Chief Nursing Officer, Office of the National Coordinator for Health Information Technology, throughout our explorations.

\section{References}

1. O’Brien A, Weaver C, Settergren TT, Hook ML, Ivory CH. EHR Documentation: The Hype and the Hope for Improving Nursing Satisfaction and Quality Outcomes. Nurs Adm Q. 2015 Oct-Dec;39(4):333-9. doi: 10.1097/NAQ.0000000000000132.

2. Effken, JA, Weaver, C. A. Spring cleaning-The informatics version. Online J Nurs Inform. In press. 
